\title{
Acquisition and extinction in the presence of the same intertrial stimuli: The effects of partial reward*
}

\author{
D. R. ZIFF, R. A. DICKERMAN, and K. A. LOWERY \\ University of Missouri-St. Louis, St. Louis, Missouri 63121
}

\begin{abstract}
Two groups of rats $(\mathrm{N}=11)$ were trained at two trials a day for 16 days in Phase $\mathrm{I}$ and 13 days in Phase II. Responses on Trial 1 were always rewarded in both phases. Percentage of reward (50\% vs $100 \%)$ was varied on Trial 2 of each day of Phase I. Trial 2 on each day of Phase II was never rewarded. A partial reward effect (PRE) was observed on Trial 2 of Phase II. The implications of the results for intertrial explanations of the PRE were discussed.
\end{abstract}

Many interpretations (e.g., Amsel, 1962; Capaldi, 1971) attribute the partial reward extinction effect (PRE) to a generalization decrement factor. Such theories assume that the change from internal stimuli conditioned during acquisition to those present during extinction is greater for consistent reward than for partial reward.

One line of research has attempted to determine whether or not the PRE can be eliminated by eliminating one or another source of internal stimulus produced generalization decrement. For example, Weinstock's $(1954,1958)$ failure to eliminate the PRE when trials were spaced by $24 \mathrm{~h}$ has been interpreted (Amsel, 1962) as a disconfirmation of the Hull-Sheffield hypothesis (Hull, 1952; Sheffield, 1949), which proposes internal stimulus traces of relatively short duration. And, for example, Gray (1969) interpreted his elimination of the PRE with sodium amytal as support for a frustrative view of the internal stimuli underlying the PRE.

Capaldi $(1967,1971)$ has proposed that the internal stimuli responsible for the PRE (be they frustrative or not) are the result of an intertrial mechanism. According to this view, reward and nonreward produce internal stimulation which may be carried over from one trial to the next in the form of memories.

The present experiment was run to determine whether or not the PRE would be eliminated by eliminating intertrial sources of generalization decrement. For both a partial reward group (Group PR) and a consistent reward group (Group $C R$ ), acquisition and extinction were studied in the presence of the same intertrial stimuli. This was achieved by a two trial per day procedure which was used throughout acquisition and

\footnotetext{
*'This investigation was supported in part by a summer research fellowship from the University of Missouri-St. Louis to the first author and by a grant of the College Work-Study Program Title IV-C of the Higher Education Act, 1965, to the second au thor.
}

extinction. The first trial of each day was rewarded, and the second trial of each day followed the first by $30 \mathrm{sec}$. In this manner, $S$ could be run in the presence of the same intertrial stimuli $\left(S^{R_{30}} \mathrm{sec}\right.$ in Capaldi's terminology) once each day on Trial 2. During acquisition, Group PR received $50 \%$ partial reward of $\mathrm{S}^{\mathrm{R}_{30}} \mathrm{sec}$ (Trial 2 was rewarded on a $50 \%$ schedule) and Group CR received consistent reward of the instrumental reaction in the presence of $S^{R_{30}} \mathrm{sec}$ (Trial 2 was consistently reinforced). Following acquisition, both groups received extinction of the instrumental response in the presence of $S^{R_{30}} \mathrm{sec}$ (Trial 2 was consistently nonrewarded).

\section{METHOD}

The Ss, 22 male albino rats purchased from the Holtzman Co., Madison, Wisconsin, were 90 days old upon arrival at the laboratory.

The apparatus was a gray wooden runway, 72 in. long, 4 in. wide, with 9-in.-high sides, covered with hardware cloth. A 10-in.-long floor treadle, suspended over a microswitch, constituted the initial portion of the alley, and when the treadle was depressed by the rat, whose front paws were always placed at its extreme forward edge, the first clock $(.01 \mathrm{sec})$ was started. Interrupting a photobeam $4.5 \mathrm{in}$. beyond the treadle stopped Clock 1 (start time) and started Clock 2. Interrupting a photobeam 42 in. beyond the first photobeam stopped Clock 2 (run time) and started Clock 3 . Interrupting a photobeam 12 in. beyond the second photobeam and in from the front edge of a 1-in.-diam foodcup stopped Clock 3 (goal time). A guillotine door $15 \mathrm{in}$. from the end of the alley was used to confine $S$ in the goal area.

Two days after arrival at the laboratory, Ss were placed on a daily feeding schedule of $12 \mathrm{~g}$ of Wayne Lab-Blox with ad lib access to water, and pretraining, which consisted of 7 days of handling and adaptation to $.045-\mathrm{g}$ Noyes food pellets, was started. On the last day of pretraining, each $S$ received two rewarded goalbox placements.

On the day following pretraining, the first of two phases of experimental training was started. On every day of experimental training, each $S$ received two trials, the first always being rewarded and the second following the first by $30 \mathrm{sec}$ of home cage confinement. For Phase I, which lasted 16 days, Ss were divided randomly into two equal groups $(\mathrm{N}=11)$ which differed 


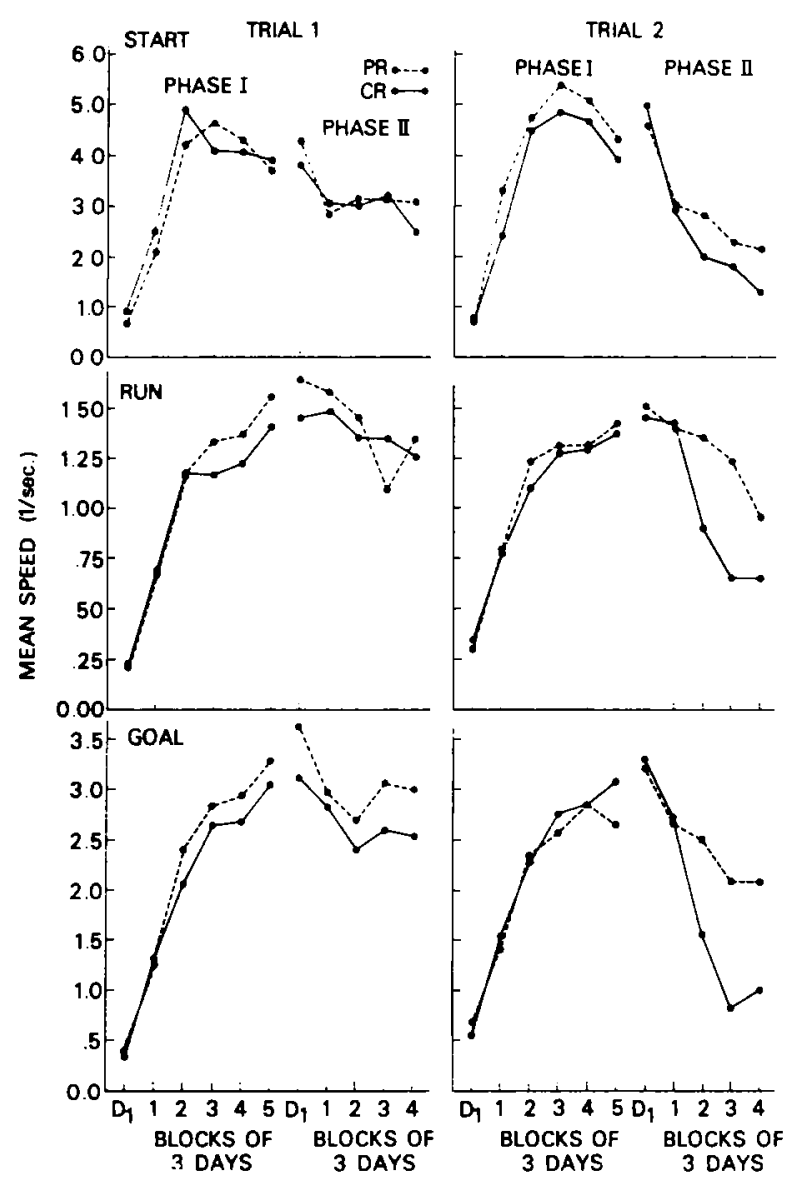

Fig. 1. Mean Trial 1 and Trial 2 start, run, and goal speeds on the first day of each phase with subsequent days of each phase plotted in blocks of three.

in the Trial 2 schedule of reward. One group (Group CR) received a consistent reward schedule on Trial 2 of Phase 1; the other group (Group PR) received a $50 \%$ partial reward schedule on Trial 2 of Phase I. The partial reward schedule of rewards (R) and nonrewards (N) was RNRNNRRNNNRRRNNR. During Phase II, which lasted 13 days, all Ss received consistent nonreward on Trial 2.

On all rewarded trials and placements, $S$ was allowed to eat 12 .045 -g Noyes pellets which had been placed in the goalcup. On all nonrewarded trials, $\mathrm{S}$ was confined in the goalbox without food for $30 \mathrm{sec}$. Ss were fed their daily ration approximately $30 \mathrm{~min}$ following training.

\section{RESULTS}

Start, run, and goal times were converted to speed scores $(1 / \mathrm{sec})$. Figure 1 presents mean Trial 1 and Triai 2 start, run, and goal speeds for the first day of each phase, with the subsequent days of each phase plotted in blocks of three.

\section{Phase I}

A mixed 2 by 2 by 16 analysis of variance with percentage ( $P R$ vs $C R$ ), trials (Trial 1 vs Trial 2), and days (the 16 Phase I days) run on the daily mean Phase I speeds in each alley section showed simply that:
(1) response speed increased over days; (2) response speed in the start section on Trial 1 was higher than on Trial 2; (3) response speed in the run and goal was higher on Trial 1 than on Trial 2 early but not late in Phase I: and (4) percentage did not reliably affect responding except in the goal section, where a Percentage by Trials interaction was found. The corresponding effects were found to be significant $(p<.05)$ : $(1)$ days (start $F=$ 9.43 , run $F=21.78$, goal $F=17.17$, df $=15 / 300$ ), (2) trials (start $F=12.60, \mathrm{df}=1 / 20$ ), (3) Days by Trials (run $F=2.03$, goal $F=2.29$, $\mathrm{df}=15 / 300$ ), and (4) Percentage by Trials (goal $F=9.44$, df $=1 / 20$ ). Mixed 2 by 13 analyses of variance with percentage and days performed on the Phase I Trial 1 and Trial 2 data separately indicated no significant main effects of percentage.

\section{Phase II}

A 2 by 2 by 13 mixed analysis of variance with percentage (PR vs CR), trials (Trial 1 vs Trial 2), and days (the 13 Phase II days) run on the daily mean Phase II speeds in each alley section supported the following: (1) Responding was slower on Trial 2 than on Trial 1; (2) responding decreased over days; (3) the decrease in responding over days was larger on Trial 2 than on Trial 1 ; (4) PR produced faster responding than $\mathrm{CR}$; (5) the PR vs CR difference was larger on Trial 2 than on Trial $1 ;(6)$ the PR vs CR difference increased over days; and (7) the increase in the PR vs CR difference over days was larger on Trial 2 than on Trial 1. The corresponding effects were found to be statistically significant $(p<.05)$ : (1) trials (start $F=$ 51.75 , run $F=162.81$, goal $F=74.66, d f=1 / 20)$, (2) days (start $F=9.43$, run $F=21.78$, goal $F=17.17$, $\mathrm{df}=12 / 240$ ), (3) Trials by Days (start $F=4.48$, run $F=$ 5.70 , goal $\mathrm{F}=7.82, \mathrm{df}=12 / 240$ ), (4) percentage (run $\mathrm{F}$ $=7.68$, goal $\mathrm{F}=9.65, \mathrm{df}=1 / 20$ ), (5) Percentage by Trials (start $F=4.44$, run $F=20.03$, goal $F=6.54, d f=$ $1 / 20$ ), (6) Percentage by Days (start $F=2.01$, run $F=$ 3.31 , goal $\mathrm{F}=2.78, \mathrm{df}=12 / 240$ ), and (7) Percentage by Days by Trials (run $F=3.87$, goal $F=2.08$, $\mathrm{df}=$ 12/240).

Mixed 2 by 13 analyses of variance with percentage and days performed on the Trial 1 and Trial 2 data separately indicated that $P R$ produced faster responding than did $C R$ on Trial 2 (run $F=13.43, p<.005$, goal $F$ $=15.18, \mathrm{p}<.001, \mathrm{df}=1 / 20$ ), but not on Trial 1 (all ps $<.10$ ), and a Percentage by Days interaction occurred in all alley sections on Trial 2 (start $F=1.99, p<.05$; run $F=3.94, p<.001$; goal $F=3.32, p<.001, d f=$ $12 / 240$ ), but only in the run section on Trial 1 (run $F=$ $2.40, \mathrm{p}<.005)$

\section{DISCUSSION}

The major finding of the present study was that, relative to the consistent reward control, partial reward 
on Trial 2 during Phase I increased resistance to extinction on Trial 2 during Phase II. This PRE was demonstrated both in reliable group differences in mean Phase II performance and in reliable Groups by Days interactions. The PRE occurred even though Trial 1 of each day of each phase was always reinforced. It will be argued that these data have particular significance for current intertrial explanations of the PRE such as Capaldi's $(1967,1971)$ sequential hypothesis.

Capaldi (1971) has proposed that the PRE as well as other phenomena can be understood if one assumes the existence of intertrial stimuli in the form of memories. According to this view, $S$ can, on any given trial, remember the goal event of the previous trial. Relative performance of $P R$ and $C R$ Ss during extinction is assumed to be related to the relative amount of generalization from memories conditioned during acquisition to those carried over from trial to trial during extinction. This explanation is satisfactory for most previous investigations of the PRE, because in most previous studies the extinction memory has been that of nonreward, a memory that has been previously conditioned for PR Ss but not for CR Ss.

This explanation cannot account for the observation of a PRE under the conditions of the present study. In the present study, each extinction trial was directly preceded by a rewarded trial, and therefore the usual extinction memory of nonreward would have been replaced by the memory of reward, a memory that had been directly conditioned in both PR and CR Ss during acquisition. Indeed, if there were any differences in generalization from acquisition to extinction, one would have expected the $\mathrm{CR}$ group to perform more strongly during extinction because the extinction memory-the memory of reward-had been conditioned more frequently during acquisition in the CR Ss. In summary, the finding that the PRE was not eliminated by eliminating memory-produced generalization decrement poses a problem for sequential theory.

Within sequential theory, any one of a number of possible assumptions may be made to account for the present finding of a PRE. A general tack is to assume that for any one of a variety of reasons, the extinction memory was not the memory of reward but rather the memory of nonreward, a memory conditioned during acquisition for PR Ss but not CR Ss. First, it could be assumed that the single reward on Trial 1 on each day of Phase II may not have been sufficient to completely replace the memory of nonrewards occurring on previous days. Or second, it could be assumed that Trials 1 and 2 differ in some unspecified way such that reward on Trial 2 of each day of Phase II may not have been sufficient to completely replace the day of Phase II remembers not reward from Trial 1, but nonreward from Trial 2 of the previous day. Or third, it could be assumed that one memory evokes another in such a way that on Trial 2, a first memory, the memory of reward from Trial 1, comes to evoke a second memory, the memory of nonreward, because nonreward was the last goal event to occur in the presence of first memory.

Phase II of the present study may also be viewed as an intertrial stimulus discrimination task. Since Trial 1 was always preceded by a nonrewarded trial and an ITI of 23-24 $\mathrm{h}$ and Trial 2 was always preceded by a rewarded trial and an ITI of only $30 \mathrm{sec}$, one should expect the intertrial stimuli carried over to these trials to differ considerably. Thus, it is not surprising that during Phase II, when Trial 1 was always rewarded and Trial 2 always nonrewarded, Ss learned to respond discriminatively. This intertrial stimulus discrimination was learned more rapidly by Group CR than by Group PR. Closely parallel results have been reported previously by Amsel and Ward (1965), who found that a brightness discrimination was learned more rapidly following CR than following PR.

Amsel and Ward (1965) explained their findings of a PRE in brightness discrimination within frustration theory (Ansel, 1958, 1962). In this explanation, Amsel and Ward assume that through classical conditioning one brightness, say black, comes to elicit $r_{g}-s_{g}$ and the second brightness, say white, comes to elicit $\mathrm{r}_{\mathrm{f}}-\mathrm{s}_{\mathrm{f}}$. The present finding of a PRE in intertrial stimulus discrimination could be explained if one assumed that intertrial stimuli can enter into the classical conditioning of $r_{g}-s_{g}$ and $r_{f}-s_{f}$ just as has been assumed previously in the case of apparatus cues such as brightness. Assigning to intertrial stimuli a position in frustration theory the same as that currently played by apparatus cues could hardly be considered a minor theoretical change. As noted by Capaldi (1967), theories which assume that intertrial stimuli play an important part in controlling behavior are likely to identify quite different variables than theories which do not do so.

\section{REFERENCES}

Amsel, A. The role of frustrative nonreward in noncontinuous reward situations. Psychological Bulletin, 1958, 55, 102-119.

Amsel, A. Frustrative nonreward in partial reinforcement and discrimination learning: Some recent history and a theoretical cxtension. Psychological Review, 1962, 59, 306-328.

Amsel, A., \& Ward, J. S. Frustration and persistence: Resistance to discrimination following prior experience with the discriminanda. Psychological Monographs, 1965, 79(4, Whole No. 597).

Capaldi, E. J. A sequential hypothesis of instrumental learning. In K. W. Spence and J. T. Spence (Eds.), The psychology of learning and motriation. Vol. 1. New York: Academic Press, 1967. Pp. 67-156

Capaldi, E. J. Memory and learning: A sequential viewpoint. In W. K. Honig and P. H. R. James (Eds.), Animal memory. New York: Academic Press, 1971

Gray, J. A. Sodium amobarbital and effects of frustrative nonreward. Journal of Comparative \& Physiological Psychology, 1969,69, 55-64.

Hull, C. L. A behalior system. New Haven. Conn: Yalc University Press, 1952

Sheffield, V. I: Extinction as a function of partial reinforcement and distribution of practice. Journal of Lxperimental Psychology, 1949, 39, $511-526$. 
Weinstock. S. Resistance to extinction of a running response following partial reinforcement under widely spaced trials. Journal of Comparative \& Physiological Psychology, 1954, 47, 318-322.

W'einstock. S. Acquisition and extinction of a partially reinforced running response at a 24-hour intertrial interval. Journal of Experimental Psychology, 1958. 56. 151-158.

(Received for publication June 5. 1972; revision received September 12, 1972.) 